UDK 782.91 Puliti:821.131.1-1

Gianmario Merizzi

Mestni glasbenobibliografski muzej, Bologna

Civico Museo Bibliografico Musicale, Bologna

\title{
Le fonti poetiche delle mascherate di Gabriello Puliti*
}

\begin{abstract}
Sommario
Rimasta fino ad ora ampiamente lacunosa, la ricerca delle fonti poetiche della Ghirlanda odorifera di Gabriello Puliti (1612), sirivela invece particolarmente fruttuosa dal momento che la quasi totalità dei testi risulta appartenere ad una produzione d'autore, le Mascherate del poeta popolare bolognese Giulio Cesare Croce (1603), oppure preesistere nelle fonti a stampa di musica vocale profana pubblicate a Venezia nei primi anni del secolo XVII.

Abbiamo così da un lato la possibilità di illustrare un caso, davvero raro per il repertorio musicale minore (non madrigalistico), di utilizzo intensivo di una raccolta poetica d'autore; dall'altro possiamo identificare musicisti e fonti musicali che funsero da modello per Puliti al momento della scelta dei testi da intonare, scoprendo per esempio, accanto ai nomi già noti di Orazio Vecchi e Adriano Banchieri, quello di Filippo Nicoletti con le sue Villanelle del 1604. L'articolo indaga il lavoro di selezione e di intervento sui testi operato, si presume, dal musicista stesso, e rileva accanto agli emendamenti intenzionali anche errori o fraintendimenti nella trascrizione dei testi originali.

Infine le date di edizione delle fonti inducono a ipotizzare una datazione della composizione della Ghirlanda più vicina all'inizio del secolo mentre l'evidenza della non originalità dei testi poetici e della loro provenienza da autori e fonti padane (perlo più emiliane) esclude la
\end{abstract}

* È necessario che denunci e giustifichi preventivamente i limiti di questo articolo. La ricerca che mi vede attualmente impegnato riguarda i rapporti di Giulio Cesare Croce con la musica. Nel corso di quell'indagine è emerso il dato, di indubbio interesse sia per chi si occupi del poeta sia per chi si occupi del compositore, riguardante le intonazioni dei testi di alcune mascherate del bolognese da parte di Gabriello Puliti. Avrei comunicato tale scoperta nell'ambito dello studio sul Croce, di cui conto di pubblicare prossimamente i risultati, se, nel corso di un incontro del tutto fortuito, la dottoressa Metoda Kokole del Muzikološki Inštitut di Ljubljana non mi avesse messo al corrente del progetto di edizione delle opere di Puliti, chiedendomi di comunicare tempestivamente le informazioni in mio possesso e prodigandosi per ottenere lo spazio di questa pubblicazione. Il poco tempo a disposizione non ha consentito che io approfondissi le mie conoscenze su Puliti né che mi occupassi degli aspetti più strettamente musicali dell'argomento, che qui si troveranno deliberatamente tralasciati. Ho invece preferito cercare di completare la panoramica delle fonti letterarie utilizzate da Puliti scoprendo alcuni legami interessanti che andranno approfonditi anche dal punto di vista musicale. Mi auguro che, nonostante tali limiti, questo contributo possa risultare utile ai curatori dell'edizione delle opere di Puliti e alla conoscenza di questo interessante compositore. Ringrazio infine la dottoressa Kokole per l'occasione offertami e per il prezioso aiuto fornito. 


\section{MUZIKOLOŠKI ZBORNIK • MUSICOLOGICAL ANNUAL XXXVI}

possibilità di leggere questa raccolta di mascherate come testimone, seppur indiretto, di espressioni culturali istriane.

L'articolo si occupa in modo pressoché esclusivo della componente letteraria tralasciando l'indagine dei rapporti tra i modelli e le realizzazioni musicali di Puliti. In appendice l'edizione dei testi di Giulio Cesare Croce intonati da Puliti.

Nei primi anni del Seicento il bolognese Giulio Cesare Croce dà alle stampe una raccolta poetica di ventisette mascherate "dalle quali - così recita il frontespizio, - pigliandosi l'inventioni si possono fare concerti dilettevoli, et gratiosi, per passa tempo il Carnevale". Quasi un decennio più tardi Gabriello Puliti darà prova di avere accolto tale invito pubblicando l'intonazione musicale di otto di quei testi nella sua Ghirlanda odorifera di varij fior tessuta, cioè mascherate a tre voci, libro primo, Venezia, Giacomo Vincenti 1612 e dedicandola a Tranquillo Negri da Venezia il 10 gennaio, agli esordi del periodo carnevalesco.

Per quanto riguarda il repertorio vocale profano minore (non madrigalistico) il fatto riveste carattere di eccezionalità sia per l'utilizzo così intensivo di un repertorio letterario d'autore (sia pure autore popolaresco), sia perché ci offre la rara opportunità di documentare, con sufficiente probabilità, il passaggio dei testi poetici dalla stampa letteraria all'edizione musicale. ${ }^{1}$ Nemmeno nell'ambito poetico e musicale più aulico e raffinato del madrigale, in cui il ricorso a componimenti poetici d'autore è assai più consueto, Puliti mostrò mai di prediligere un poeta in maniera tanto consistente. ${ }^{2}$ L'attenzione e l'abilità del musicista nella selezione di testi poetici 'a tema' erano già emerse in occasione del suo secondo libro di madrigali (Baci ardenti, Venezia 1609) per il quale Puliti non selezionò più di 5 testi dalla prima parte delle Rime di Giovan Battista Marino (1602), assieme a Guarini il poeta in assoluto più utilizzato dai madrigalisti in quegli anni. ${ }^{3}$ Una piccola rivincita per l'umile cantastorie bolognese cui l'autore dell' Adone aveva voluto indirizzare alcuni versi invero poco lusinghieri. ${ }^{4}$

Gli studi finora dedicati alla Ghirland $a^{5}$ non si sono in effetti preoccupati di ricercare sistematicamente le fonti dei testi poetici intonati, finendo più o meno implicitamente

1 Si veda a riguardo Concetta Assenza, La canzonetta dal 1570 al 1615, Lucca, Libreria musicale italiana 1997, pp. 103-104, $129 \mathrm{e}$ in generale il capitolo relativo al repertorio poetico che affronta varie tematiche coinvolte nel presente articolo.

Come è noto la nostra conoscenza della produzione di Puliti è lacunosa: manca all'appello almeno il primo libro di madrigali a 5 voci, riportato dal catalogo del libraio fiorentino Giunta, edito nel 1604, che li qualifica come madrigali apastoralin (vedi $O$. Mischiati, Indici, cataloghi e avvisi degli editori e librai musicali italiani dal 1591 al 1798, Firenze, Olschki 1984, p. 121.) I. Cavallini (Fortuna del madrigale in Istria, lo cito dalla riedizione in Id., Musica, cultura e spettacolo in Istria tra '500 e '600, Firenze, Olschki 1990, pp. 75-93) ne inferisce una scelta di testi di Guarini o Sannazzaro (p. 91). Un esemplare dell'opera è attestato nell'inventario dei beni musicali dell'arciduca Siegmund Franz di Innsbruck redatto nel 1665 (cfr. Franz Waldner, Zwei Inventarien aus dem XVI. und XVII. Jahrhundert über hinterlassene Musikinstrumente und Musikalien am Innsbrucker Hofe, "Studien zur Musikwissenschaft" IV (1916), pp. 128-147 : 143. È verosimile che parte della raccolta ducale provenisse da Ferrara e abbia in seguito preso la via di Vienna.

3 Sempre nei Baci ardentisi individuano inoltre due poesie di Francesco Contarini, due di Pietro Petracci, una di Valerio Buratini, e una di Matteo Piacentini; negli Armonici accenti (1621) una poesia di Angelico Michelangelo, una di Ottavio Rinuccini, una di un poeta sotto pseudonimo di Esone Accademico Trasformato, e i due madrigali attribuiti nella stampa a Ottonello del Bello e Giambattista Brati. Per l'attribuzione dei testi ho potuto consultare il costituendo Repertorio della poesia italiana musicata dal 1500 al 1700 curato da Angelo Pompilio e Antonio Vassalli. Il repertorio non riporta tuttavia le Mascherate di Giulio Cesare Croce.

Si legga il sonetto LXII della Murtoleide.

Mi riferisco a: Ennio Stipčević, Maskerate Gabriella Pulitija, "Sveta Cecilija” LIII (1983), pp. 60-62, 83-85; LIV (1984), pp. 9-10; Ivano Cavallini, Feste e spettacoli in Istria tra Cinque e Seicento ele mascherate a tre voci di Gabriello Puliti, ,Il flauto dolce" XIV/XV (1986), pp. 3-15, poi rivisto e ripubblicato in "Arti Musices" XXI (1990), pp. 99-125 e in Id., Musica, cultura e spettacolo ..., cit., pp. 165-195. Le citazioni che seguono fanno riferimento a quest'ultima edizione. 
per investire il compositore di responsabilità anche nella confezione dei testi poetici. Certo, la paternità crocesca dei testi non avrebbe potuto emergere che da uno studio sul versante delle fonti poetiche; ma per quasi tutte le altre rime della raccolta l'utilizzo dei repertori musicali correnti ${ }^{6}$ è sufficiente a rivelarne almeno la preesistenza nel repertorio della musica vocale, consentendo di tracciarne la provenienza da alcune specifiche edizioni e di valutare gli interventi che (probabilmente) il musicista stesso operò per adattare i versi alle proprie esigenze.

Il seguente schema, che verrà di seguito illustrato, sintetizza la situazione complessiva che è stato possibile accertare:

\section{Tab. 1}

G. Puuti, Ghirlanda, 1612 (dalla tavola) Fonti letterarie

1 Vedove sconsolate

2 Donne mal maritate

3 Cantori sventurati

4 Spose contente

5 Villani ch'hanno legata la creanza

6 Dottor Gratian da Francolin

7 Amanti

8 Spazza Camin

9 Pantaloni inamorai

10 Gobbi

11 Ortolani

12 Cavaliero assaltato d'Amore

13 Poeti Bischizzanti

14 Soldati svalegiati

15 Amor di cortegiana incostante

16 Todeschi

17 Astrologi

18 Orbi, e Ciechi affatto

19 Falliti

Non musicata)

20 Humoristi

21 Matti

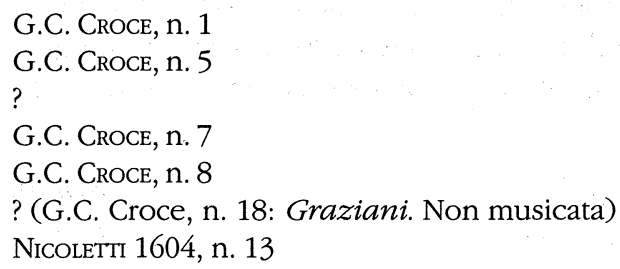

Partiremo dall'esame dei testi di 'tradizione musicale' lasciando per ultimo l'esame della fonte letteraria crocesca.

Tre soli testi restano privi di tradizione attestabile. Per uno di essi, la 'trasmutazione' di Ancor che col partire, madrigale di Alfonso d'Avalos reso celeberrimo dall'intonazione di

Si sono consultati: E. Vogel, A. Einstein, F. Lesure, C. Sartori, Bibliografia della musica italiana vocale profana pubblicata dal 1500 al 1700 , Pomezia-Genève, Staderini-Minkoff 1977; H. B. Lincoln, The Italian madrigal and related repertories. Index to printed collections, 1500-1600, New Haven-London, Yale University Press 1988; Il repertorio vocale profano nelle raccolte a stampa del secolo XVII, a cura di M. Mangani, Roma, Torre d'Orfeo 1997. 
Cipriano de Rore, è in realtà possibile indicare un ventaglio di modelli sia come genere letterario (in cui tra l'altro Giulio Cesare Croce non mancò di cimentarsi generosamente), sia nella sua trasposizione musicale inaugurata da Orazio Vecchi nell' Amfiparnaso proprio con questo testo (e con la musica di Rore) e poi applicata da Banchieri a questo e ad altri celebri madrigali nelle sue 'commedie harmoniche' a tre voci. ${ }^{7}$ Ma Ancor che col partire, forse per l'esemplarità conferitagli dall'invenzione di Vecchi, resta il testo più utilizzato per le 'trasmutazioni' musicali; e se i precedenti di Vecchi e Banchieri risultano di particolare importanza per il ruolo privilegiato che questi musicisti occupano nell'orizzonte ispirativo di Puliti, non va scordato il contributo di Andrea Gabrieli, ${ }^{8}$ principale titolare di una delle più importanti sillogi di mascherate musicali ${ }^{9}$ che Puliti, come si dirà, quasi certamente conosceva.

Di Banchieri, Puliti riprende l'Intermedio di Spazzacamini, nella versione leggermente modificata dalla seconda e di gran lunga più diffusa edizione della Pazzia senile che si continuava a ristampare in quegli anni. ${ }^{10}$ Puliti modifica il verso finale in modo da ottenere una ripresa (anche musicale) del distico iniziale, inesistente nel modello.

Puliti, p. 8

Spazza camin

Olà bella brigada

Hù hù spazza cami(n)

Nù sem dalla vallada

Dov nasc'i bon facchi

Nù sgurarem e fregarem

Coù smozzigù i vos canù*

E farem prest Però con quest

sia parrechia formai e pa

e tutto quest farem per un Carli

Olà bella brigada

Hù hù spazza camì.
Banchieri (1599, n. 13)

Intermedio terzo di spazzacamini

O la bella brigada,

Hù hù spazza camì.

$\mathrm{Nu}$ sem dalla vallada

Dov nass i bon fachì.

Nu sgurarem, e fregarem

Coi smozegù* i vos canù

$\mathrm{E}$ farem prest però con quest

Sia parechià furmai e pà.

E tutto quest farem per un carlì.

Bella brigada hù, hù spazzacami**.

* 1598: fureghu

** 1598: Bella brigada, sem spazzacami.

Ivano Cavallini ha alacremente illustrato i molteplici legami della produzione di Puliti con l'opera musicale banchieriana. Nel caso della Ghirlanda, tuttavia, il fatto che il prolifico musicista bolognese sia, accanto a Puliti, uno degli ultimi musicisti a coltivare non

7 Il rilievo è già in E. Stipčević, Maskerate ..., cit. pp. 84-85. Le trasmutazioni di Banchieri si trovano nello Studio dilettevolee nel Metamorfosi musicale, entrambi riscritture a 3 voci dell' Amfiparnaso di Vecchi, e nella Pazzia senile da cui proviene uno dei testi intonati da Puliti nella Ghirlanda.

8 Si tratta della giustiniana Ancorche col partire me sento sgagiolire, in Greghesche et Iustiniane a 3 voci, libro I, Venezia 1571.

9 Mascherate di Andrea Gabrieli et altri autori eccellentissimi a 3, 4, 5, 6 e 8 voci, Venezia 1601. Ne resta un solo esemplare,

purtroppo incompleto.

10 La prima edizione dell'opera è del 1598; la seconda è del 1599; si conoscono ristampe nel 1601, 1604, 1607, 1611, 1617, 1621. 
occasionalmente il genere della mascherata polifonica, ${ }^{11}$ fa sì che, a prescindere da un effettivo legame diretto, non sia difficile trovare affinità tra la produzione dei due autori nell'ambito di un genere che affonda le radici in un diffuso sostrato culturale, rituale, letterario e teatrale. La mascherata dei Soldati svalegiati ne è un esempio: Cavallini ${ }^{12}$ coglie nel segno nel segnalare il legame con I poveri soldati venuti d'Ungheria che chiude la Barca di Venezia per Padova di Banchieri (1605), ma in questo caso il legame è probabilmente indiretto poiché entrambi i musicisti ricavano il testo o il 'soggetto' (nel caso di Banchieri, che scrisse buona parte dei testi delle sue composizioni) dalla poesia di Giulio Cesare Croce che a sua volta lo attinse dalla tradizione (e forse in parte dalla realtà della strada). Ciò non toglie che il precedente di Banchieri possa avere incoraggiato Puliti ad utilizzare quel testo o addirittura l'intera raccolta delle mascherate poetiche del Croce.

Ancor più di Banchieri, Orazio Vecchi deve aver costituito un importante riferimento per Puliti al momento della scelta dei testi da intonare. Nella già citata raccolta miscellanea di mascherate intestata ad Andrea Gabrieli $(1601)^{13}$ Orazio Vecchi è quantitativamente il secondo autore presente, con 5 composizioni che non si ritrovano in altre fonti conosciute. ${ }^{14}$ Tra queste Puliti riprende il testo della Mascherata d'hortolani (n. 11 della Ghirlanda) e quello della Mascherata d'astrologi(n. 17). Mentre il primo resta identico all'originale, fatte salve le consuete varianti ortografiche, il secondo presenta delle omissioni. Manca interamente la seconda strofa, contenente un riferimento alla città di Correggio dove Vecchi fu attivo dal 1586 al 1593. Esiste però un'ulteriore spiegazione, più interessante, per questa omissione: qui (come, con più evidenza, nel brano n. 15 , Amor di cortegiana incostante) Puliti opta per una intonazione di carattere madrigalistico dove la musica ricerca un rapporto espressivo (anche visivo, notazionale) univoco col testo e non si presta dunque ad intonare più strofe. ${ }^{15}$ Forse involontaria è invece l'omissione del sesto verso della prima strofa, omissione che crea una lacuna nella sintassi del periodo conclusivo e una conseguente incertezza semantica. Non possediamo tutti i libri parte della stampa del 1601 per verificare appieno questa ipotesi, ma è possibile che Puliti trascrivesse il testo dalla parte del Basso ove quel verso, intonato dalle altre voci, viene in effetti a mancare.

11 Se concepiamo la mascherata partendo dalla sua componente rituale e rappresentativa che è per tradizione una manifestazione collettiva essa appare per vocazione un genere polifonico il cui declino è segnato dall'affermazione della monodia accompagnata. "Noi siamo" è il predicato tipico di esordio delle mascherate, cui si aggiunge spesso, prima dell'indicazione della categoria sociale, il numero (tre, quattro, cinque) dei mascherati che ha naturalmente un rapporto diretto col numero della voci della composizione polifonica.

Op. cit., p. 191.

13 Vedi nota 9

14 Sono presenti inoltre Geminiano Capilupi con due composizioni e Ippolito Camaterò con una.

15 Si veda quanto osserva I. Cavallini, op. cit., pp. 187-188, proprio per questi due brani in relazione all'uso di artifici mensurali. 
Puliti, p. 17

Astrologi

Astrologi noi siam donne amorose

Che col nostro sapere

apriam le porte più remote $e$ ascose

delle celesti sfere

E dell'humane cose

Che tosto che miriamo

Il vostro aspetto e la vostra natura

Dir vi saprem ò buona ò ria ventura.

\author{
O. Vecchi (1601) \\ Mascherata d'Astrologhi à 6 \\ Astrologhi noi siam Donne amorose \\ Che co'l nostro sapere \\ Apriam le parte (porte) più remote e ascose \\ De le celesti Sfere \\ E de l'humane cose \\ Voi vedrete in effetto \\ Che tosto che miriamo il vostr'aspetto \\ e la vostra natura \\ Dir vi saprem'ò buona ò ria ventura. \\ Noi vi farem toccar proprio con mano \\ Le materie più sode, \\ Qual sia COR Regio, e qual sia cor villano ...
}

Due altri testi della Ghirlanda provengono con probabilità da opere Orazio Vecchi. Il testo della mascherata n. 12, Cavaliero assaltato d'Amore ("Amor con ogni imperio e gran possanza") lo si rintraccia già in due edizioni della fine degli anni Sessanta del Cinquecento: Delle napolitane di G. B. Villanova libro secondo a 3 voci (Milano, 1568) e Primo libro della raccolta di napolitane a 3 voci di diversi eccellentissimi musici (Venezia, 1570) per la messa in musica di Bernardino Franzosino. Nella fonte più antica il testo consta di sette strofe (terzine). Le sole prime 4 hanno un refrain nel terzo verso ("A suon di trombe e di tamburi et armin) e questo è forse il motivo per cui le tre rimanenti non si ritrovano nelle edizioni successive. ${ }^{16}$ Non possiamo escludere che Puliti conoscesse una di queste due edizioni ma è di gran lunga più probabile che conoscesse la terza fonte a noi nota di quel testo, cioè il Secondo libro delle canzonette a 4 voci di Orazio Vecchi, stampato la prima volta nel 1580 e ristampato nel 1582, 1585, 1595 e ancora nel 1602. La nostra supposizione è avvalorata dal fatto che la medesima raccolta di canzonette contiene anche il testo della mascherata successiva nella sequenza della Ghirlanda (n. 13, Poeti bischizzanti: "O donna ch'al mio danno i ciel ti denno") che non ritroviamo invece in alcuna altra fonte musicale o letteraria nota. Le versioni del Cavaliero assaltato d'Amore in Vecchi-Puliti sono sostanzialmente identiche e presentano significative varianti rispetto alle due edizioni più antiche:

16 Il testo si ritrova però anche nel manoscritto I-MOe C311, Arie e canzoni in musica di Cosimo Bottegari (1574), c. 51v. Vengono utilizzate le prime tre e la quinta strofa nell'edizione di Villanova. Manca quindi la quarta strofa, presente nella Ghirlanda. 


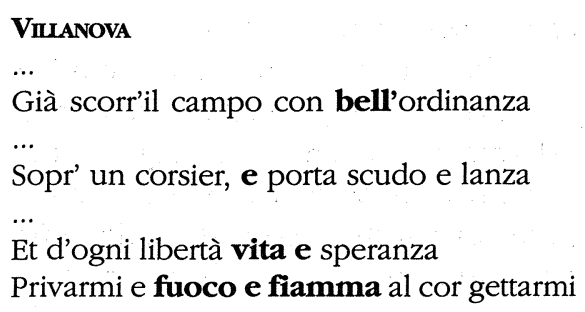

\section{Franzosino}

...

Già scorre il campo con grand'ordinanza

...

Sopra un corsier et porta scudo et lanza

...

E d'ogni libertà vita et speranza

Privarmi e fiamme e foco al cor gettarmi

Vecchi - Pulmi (tra parentesi le varianti ortografiche)

...

Già scorre il campo con grand'ordinanza

...

Sopra un corsiero porta scudo e(t) lanza

...

E d'ogni libertà d'ogni speranza

Privarmi $e(t)$ fiamme $\mathbf{e}(\mathbf{t})$ fuoco al cor gittarmi (gettarmi).

Il testo dei Poeti bischizzanti presenta varianti di rilievo nelle due terzine finali:

Puliti, p. 13

Lo spirto e [= è?] sperto e sparto e a ogni suo cenno

Vola veloce ove tua voglia el tira

D'amor amaro oime ch'io moro mira.

Se'l tuo decoro o caro e [ $=$ è?] cura al core N'ha, perch'hor non deve dire ardore Mira ch'io moro oime d'amaro amore.

\section{Vecchi}

Lo spirto esperto è sparto, e a ogni tuo cenno

Vola veloce ove tua voglia il tira

D'Amor [amaro ohime ch'io moro mira].

Se'l tuo decoro hò caro, et cura il core N'ha, perche ardire hor dir non deve ardore Mira ch'io moro ohime d'amaro Amore.

La versione utilizzata da Puliti è evidentemente corrotta: le varianti introducono errori lessicali (sperto, che potrebbe però essere forma toscana per esperto), logici (tuio invece che suo), grammaticali (viene a mancare il soggetto del verbo ha), metrici (il penultimo verso è ipometro). Di fronte a tali varianti, prima di rinunciare all'ipotesi che la stampa del Vecchi sia stata la fonte di Puliti, date le scorse considerazioni, possiamo supporre che l'astrusità di questi versi possa aver indotto errori di trascrizione, forse imputabili al musicista, forse allo stampatore.

Se Vecchi e Banchieri sono riferimenti collaudati dell'opera di Puliti, una novità interessante è invece costituita da Filippo Nicoletti con le sue Villanelle a 3 voci (Venezia, 
1604) da cui Puliti ricavò 4 dei testi intonati nella Ghirlanda (nn. 7, 10, 19, 20). Dopo avere studiato a Bologna con Giuliano Cartari, Nicoletti fu attivo a Rovigo e quindi a Ferrara, sua città natale, ove rimase fino all'anno di edizione delle villanelle, assumendo poi l'incarico di maestro di cappella in S. Lorenzo in Damaso. ${ }^{17}$ I quattro testi in questione, che non ci risultano attestati da altre fonti, restano sostanzialmente identici nella versione di Puliti, fatta eccezione per il verso iniziale del primo di essi che nell'edizione del 1604 recita: "Non favorite Amanti, quest'ingrate". Nella Ghirlanda esso è modificano in: "Non favorite queste donne ingrate". La variante è da attribuire alla rifunzionalizzazione della villanella in Mascherata d'amanti, secondo la didascalia della Ghirlanda: gli Amantinon sono più dunque i destinatari del messaggio cantato ma i suoi mittenti, i protagonisti della mascherata; da qui la necessità di modificare il verso d'esordio. ${ }^{18}$ I testi delle mascherate n. 10 e n. 19 richiamano il genere della quotation villotta, secondo la definizione di Alfred Einstein, includendo, almeno apparentemente, la citazione di una canzone o di un ritornello popolare, nel primo caso il Chiù chiùe Saldo Gobbetto, nel secondo la Fallilella. Richiami, motti, canzonature a danno dei gobbi sono noti e sono sopravvissuti fino ai giorni nostri. Si ricorda la celebre Canzone del Gobbo nan, citata nell'incatenatura del Bianchino $^{19}$ e ripresa anche in una operetta in rima attribuita a Giulio Cesare Croce, ${ }^{20}$ il cui ritornello "quan, quan" è funzionalmente e foneticamente analogo al "chiù, chiù" della nostra villanella. In ogni caso se citazione c'è questa non si riflette in modo evidente nella trascrizione musicale di Puliti che reinventa il motivi melodici nei due brani conservando piuttosto qualcosa dell'andamento ritmico di Nicoletti: chiù chiù, quale imitazione del verso del cuculo, presenta la medesima struttura ritmica nelle due versioni, ma Puliti preferisce l'intervallo di quarta a quello di terza usato da Nicoletti; i motivi che intonano Saldo gobbetto sono ritmicamente identici nei due autori, ma solo nell'ultima ripetizione l'intonazione di Puliti utilizza il tetracordo discendente (per sua natura poco identificante) che è alla base dell'episodio in Nicoletti; ${ }^{21}$ infine le intonazioni della Fallilella non condividono più di un generico procedere melismatico per quartine di semicrome. ${ }^{22}$

L'ultimo brano identificato, il n. 15 della Ghirlanda, intitolato Amor di cortigiana incostante ("Amor è fatto a punto come il mare") è attestato dai repertori in tre edizioni degli anni ottanta del Cinquecento. ${ }^{23}$ Non abbiamo elementi per tentare di identificare un'edizione in particolare quale fonte di Puliti, inoltre la distanza cronologica aumenta la probabilità di esistenza di una fonte più recente e sconosciuta. ${ }^{24} \mathrm{Ma}$ il dato importante ci

17 Nicoletti fu anche poeta. Sul frontespizio della raccolta delle sue Rime spirituali sovra la solennità del Natale, Roma 1604, egli esibisce già la qualifica di maestro di cappella nella basilica romana.

18 Altre varianti di qualche rilievo: nella medesima quartina "ch'io vi so diren diviene "che vi so diren; nel verso finale "Ch'havete poi da lor" diviene "Ch'havete poi da tor"; $n$. 13, secondo verso, "no'l canterete piùn diviene "non canterete più" ; $n$. 20, quinto verso, «Mi fa pensoso il coren diviene «Mi fa penoso il core".

19 Camillo Cieco detto il Bianchino, Opera nuova nella quali si contiene una incatenatura di villanelle, Verona 1629: "questa bizzarria / ... / Che si canta per Milan / ... / Quan, quan / Astu visto lo gobbo Nan?n

20 Le tremende bravate fatte dal Gobbo nan contra coloro che vanno gridando per Milano: Quan, quan hastu visto lo gobbo nan?, Bologna, Eredi di Bartolomeo Cochi 1621.

${ }_{21}$ Si veda la trascrizione in I. Cavallini, p. 190.

22 Come già detto, lascio comunque ad altra sede il compito di un confronto musicale approfondito.

23 Fabrice Marin Caietain, Second livre d'airs, chansons, villanelles napolitaines et espagnolles mis en musique à quatre parties, Paris, 1578; Gasparo Costa, Il secondo libro di canzonette a tre voci, Venezia, 1584; Iseppo Bonardo, Il primo libro delle napolitane a 3 voci, Venezia 1588.

24 L'edizione del Bonardo è tuttavia ancora registrata nel catalogo del libraio fiorentino Giunta del 1604, già citato. 


\section{MUZIKOLOŠKI ZBORNIK • MUSICOLOGICAL ANNUAL XXXVI}

sembra un altro: delle quattro terzine del testo originale (così come lo attestano le tre edizione citate) Puliti conserva solamente la prima. Il caso è analogo a quello già visto della Mascherata di astrologi, ma qui la scelta di una intonazione madrigalistica, quindi non strofica, è sottolineata dalla modificazione del terzo verso che nelle edizioni cinquecentesche recita uniformemente: "In un medesmo stato non stà mai" mentre nella versione di Puliti il sostantivo stato si tramuta in tempo, offrendo al compositore pretesto per gli espedienti notazionali descritti da Cavallini. ${ }^{25}$

Le stampe musicali, lo abbiamo testé toccato con mano, costituiscono il canale consueto dell'approvigionamento poetico dei compositori nei generi minori; al contrario l'utilizzo di una fonte letteraria d'autore, come già sottolineato, sembra costituire un fatto eccezionale o almeno raramente documentabile. Per questo dedicheremo particolare attenzione all'esame degli otto testi che Puliti trasse dalle rime del Croce.

Giulio Cesare Croce (San Giovanni in Persiceto 1550 - Bologna 1609), universalmente noto come poeta, scrittore e cantastorie, popolare e dialettale, autore del Bertoldo e del Bertoldino, non è ignoto agli studi musicologici. Curiosamente questa notorietà non gli deriva dalla sua effettiva attività di musicista, di cantore di piazza e (non di rado) di palazzo, solito intonare le proprie rime su un repertorio fisso di arie accompagnandosi con l'inseparabile 'lira' (una viola secondo le testimonianze iconografiche). La documentazione di questa attività è in effetti indiretta e generica, e più fruttuoso è parso finora attingere alle testimonianze musicali presenti nella foltissima e disparata produzione letteraria del Croce $^{26}$ oppure investigare i rapporti con musicisti famosi e segnatamente con Adriano Banchieri e Orazio Vecchi ${ }^{27}$.

Tra le numerose testimonianze musicali offerte dal Croce, di particolare interesse risulta qui un passo dalla Veglia carnevalesca nella quale s'introducono un bellissimo drapello di cavallieri e di dame e danzare e si sentono varij linguaggi e canzoni et in ultimo una bella mascherata d'ortolane che vendono del latte. ${ }^{28}$ La mascherata in questione è una delle tre che, in un'edizione postuma, venne aggiunta alla silloge utilizzata da Puliti (vedi oltre). I mascherati, che vagano per la città, di festino in festino, sopraggiungono al termine della veglia, organizzata in una casa privata, quando ormai aggiorna. La descrizione dell'esibizione è, purtroppo, condizionata dal mezzo poetico:

"Signora Madre!", "Che dice Laurina?", "Maschare, mascare, mirate, mirate, O come le son belle e ben ornate!"

"Venite pur inante, Ortolane galante, Ch'altro che voi per hora non ci resta Per compimento de la nostra festa."

Op. cit., p. 187.

Si veda per esempio l'esemplare saggio di W. Kirkendale, Franceschina, Girometta, and their companions in a madrigal "a diversi linguaggi by Luca Marenzio and Orazio Vecchi, "Acta musicologica", XIIV (1972), pp. 181-235, poi aggiornato e ripubblicato in Il madrigale tra Cinque e Seicento, a cura di Paolo Fabbri, Bologna, Il Mulino 1988, pp. $249-331$.

27 Croce fu anche in contatto personale con Girolamo Giacobbi, come dimostra la dedica de Il mondo alla roversa, Bologna, Eredi di G. Rossi 1605.

28 La prima edizione nota è stampata a Bologna da Bartolomeo Cochi 1620. La mia citazione è da una ristampa successiva: Bologna, Erede del Cochi, s.a. La punteggiatura è stata modernizzata per migliorare la comprensibilità del testo. 


\section{MUZIKOLOŠKI ZBORNIK • MUSICOLOGICAL ANNUAL XXXVI}

"Largo, largo Signori che bisogna,

Far luoco à queste mascare:

In la le banche e di gratia slargate

Il campo se volete, Che cantar udirete

Questi Musici rari et eccellenti

Qualche bel Madrigal, se state atenti."

Chi vuol del Latte, ò Donne,

Ecol candido, fresco, bel e sodo

Che non fa sero, o si converte in brodo

Ed è polito, è netto,

E di pecora schietto;

Gustatel col cochiaro over col dito

Che sentirete quanto è saporito.

Questo rinfresca dentro

E fa buon nutrimento,

Fa bella carne ingrassa e allegra il core

$E$ de la sete estingue il grave ardore.

Poi a un'altra virtù, se nol sapete,

Che voi voi felici se [n] prenderete.

Però se ne volete

Fatevi sotto con le pignatelle

Che vi sgocciolarem ben le scodelle.

"Signori à voi rendiam gratie infinite,

Del favor grande che fatto ci havete,

E con ver dir potiamo che voi sete

Unichi à questa etate, Che fra le mascherate

Che viste habbiamo in questa parte e in quella,

Il vanto à questa d'esser la più bella."

"Horsù l'hora è già tarda, udite i Galli,

Che cantano"...

È singolare, ed affascinante, come la raccolta di mascherate di Puliti con la sua peculiare scelta di testi venga a rinnovare i legami tra Croce e i due musicisti emiliani sù ricordati. Nonostante fossero concittadini non ci sono prove esplicite di un contatto personale tra Croce e Banchieri, ma numerosi sono i debiti del Banchieri letterato con l'opera del Croce e le citazioni che egli dedica al cantastorie. ${ }^{29}$ Consistenti invece le testimonianze di contatti personali tra Croce e Vecchi, tanto da alimentare l'ipotesi di un intervento del Croce nella redazione del testo dell' Amfiparnaso ${ }^{30}$ che costituirebbe un importante

Il caso più noto è la continuazione di Banchieri della saga bertoldesca cui aggiunge Le avventure di Cacasennoma cui si espirano in effetti altri suoi libri di novelle. L'elenco più completo, sebbene non esaustivo, è in A. Wernli, Studien zum literarischen und musikalischen Werk Adriano Banchieris, Bern und Stuttgart, Haupt 1981; in particolare pp. 44-46, 69-72.

30 Il primo a sollevare l'ipotesi è Edward J. Dent, Notes on the *Amfiparnaso" of Orazio Vecchi, "Sammelbände der internationalen Musikgesellschaft" XII (1911), pp. 330 sgg. 
precedente nell'attività di Croce quale autore di testi per musica. Sebbene tale ipotesi non abbia mai ricevuto una verifica definitiva, essa gode non solo del conforto degli indizi biografici ma anche del giudizio stilistico del più grande, e rimpianto, studioso del Croce, Piero Camporesi:

"a noi sembra che ... la presenza crocesca sia particolarmente forte nell'Amfiparnaso, ... dove almeno tutta la scena terza dell'atto terzo riteniamo si possa senza alcuna titubanza attribuire alla mano del Croce, esperto anche nel captare e riprodurre la voci e le parlate ebraico-emiliane e nel far rivivere scene di ghettom. ${ }^{31}$

La redazione di una bibliografia delle opere del Croce è una "fatica improba" che da decenni mette a dura prova la caparbietà degli studiosi. ${ }^{32}$ La causa sta nell'estrema prolificità dell'autore (il catalogo, provvisorio, conta 560 opere) $)^{33}$, nella straordinaria fortuna della sua opera, valutabile in una serie esplosiva di edizioni, ristampe e false attribuzioni, ${ }^{34}$ nella peculiare politica editoriale, funzionale ad un commercio minuto basato su piccoli opuscoli monografici (quando non su ventarole) piuttosto che su raccolte, nella dispersione delle fonti a stampa ${ }^{35}$, alcune delle quali già introvabili al tempo del Croce. Anche le Mascherate conobbero numerose edizioni la cui bibliografia contribuisce bene ad illustrare la cifra complessiva del problema. ${ }^{36}$ La prima edizione nota (fidandoci del dato cronologico desunto dalla dedica) è stampata a Venezia:

Le ventisette / MASCHERATE / piacevolissime / DEL CROCE / Dallequali [sic] pigliandosi l'inventioni / si possono fare Concerti dilet- / tevoli, \& gratiosi, per passa / tempo il Carnevale. / Novamente data in luce. / CON PRIVILEGIO. /[xil.]/ In Venetia Appresso Nicolò Polo.

fascicolo di 48 p. in $12^{\circ}$, con dedica a Berenice Gozadina Gozadini, nobile bolognese, sottoscritta dall'autore in data 20 dicembre $1603 .{ }^{37}$ A distanza di qualche giorno (sempre sulla base della datazione delle dediche) l'opera appare anche a Bologna:

31 P. Camporesi, Il palazzo e il cantimbanco, Giulio Cesare Croce, Milano, Garzanti 1994, p. 38.

32 Per gli aspetti complessivi del problema si rimanda alle indicazioni bibliografiche fornite da Rosaria Campioni, Una 'fatica improba': la bibliografia delle opere di Giulio Cesare Croce, in Libri, tipografi, biblioteche. Ricerche storiche dedicate a Luigi Balsamo, a cura dell'Istituto di Biblioteconomia e Paleografia Università degli Studi di Parma, Firenze, Olschki 1997, vol. II, pp. 399-420.

M. Rouch, Bibliografica delle opere di Giulio Cesare Croce, "Strada maestra" 17 (1984), pp. 229-272.

Così lo ricorda Banchieri nel Discorso della lingua bolognese (cito dall'edizione di Bologna 1629): “... Iuli Cesar Cros, alias dalla Lira, ... al n'hà cumpost più volum ch'an n'havè pil in barba, e tant fù gustos, chi Stampadur al fan cumponr quisi mort cmod l'èn (G.C. Croce, detto dalla Lira, ne ha scritti più volumi di quanti peli avesse nella barba e fu tanto apprezzato che gli stampatori lo fanno scrivere anche da morto).

35 Diversi autografi conserva la Biblioteca Universitaria di Bologna.

36 Un elenco non esaustivo è in Storie di vita popolare nelle canzoni dipiazza di G. C. Croce. Fame fatica e mascherate nel '500, opere poetiche in italiano con introduzione e note a cura di Monique Rouch, Bologna, CLUEB 1982, pp. 111-112. Nello stesso volume troviamo una breve presentazione dell'opera (pp. 130-132) e l'edizione di otto testi sulla base dell'edizione di Bologna 1604 (pp. 199-214). Due soli appartengono alla scelta intonata da Puliti.

37 Ho consultato l'esemplare conservato alla Biblioteca dell'Archiginnasio di Bologna. 
MUZIKOLOŠKI ZBORNIK • MUSICOLOGICAL ANNUAL XXXVI

MASCHERATE / PIACEVOLISSIME / DI GIULIO CESARE / DALLA CROCE, / Dalle quali pigliandosi l'Inventioni, si pos- / sono fare concerti dilettevoli, e gra- / tiosi, per passa tempo il / Carnevale. / [xil.] / In Bologna, presso gli Heredi di Giovanni Rossi. / M.DC.IIII. / Con licenza de' Superiori.

con la medesima dedica, datata però 5 gennaio $1604 .{ }^{38}$ Il contenuto delle due edizioni e l'ordine di presentazione delle 27 mascherate è il medesimo ma, al di là delle consuete discrepanze ortografiche, compaiono talune differenze di rilievo nel dettato di alcuni versi e nei titoli. Si coglie complessivamente una maggiore correttezza nella stampa bolognese, come se fra le due edizioni fosse intercorso un certo lavoro di revisione più che un semplice miglioramento nella cura tipografica. Una terza edizione nota rientra nei termini cronologici della Ghirlanda:

CINQUE / MASCHERATE / DEL CROCE, / Con [2] Ottave alla Siciliana, da reci- / tarsi nel tempo di Car- / nevale. / Date in luce da me Zan Scarpetta. /[xil.]/ In Modona, per lo Cassiani, 1609 / Con licenza dei Superiori ${ }^{39}$

Tuttavia essa non solo pubblica una scelta delle mascherate ma ne seleziona le strofe, omettendo per i due componimenti utilizzati da Puliti e qui inclusi (Todeschie Donne mal maritate) strofe che ritroviamo invece nell'edizione musicale.

La fortuna editoriale delle mascherate del Croce si protrae ben oltre il 1612, anno di pubblicazione dell'opera di Puliti. Per quello che ci è dato di conoscere (ed è certamente una frazione di quanto dovette passare sotto i torchi), l'anno seguente, 1613, Bartolomeo Cochi stampa due ventarole (ma è assai verosimile che non fossero le uniche) riportanti ciascuna il testo di una singola mascherata (Vedove che vanno piangendo $i$ loro mariti e Pantaloni innamorati) corredato da una xilografia raffigurante i personaggi in questione. Lo stesso Cochi ristampa la raccolta completa nel 1616, mentre nel 1621 essa è ripubblicata a Venezia presso Ghirardo Omberti. In quegli stessi anni vede la luce a Bologna una nuova edizione intitolata Le trenta mascherate piacevolissime ... data alle stampe prima dagli Eredi di Bartolomeo Cochi ${ }^{40}$ quindi da Antonio Pisarri. ${ }^{41}$ Come si desume dal frontespizio, si aggiungono tre nuovi testi sulla cui effettiva attribuzione crocesca non è qui il caso di dilungarsi. ${ }^{42}$ Un'edizione tardiva di tre soli testi, di cui uno nuovo e spurio, appare ancora nel 1661 a Spoleto presso Gregorio Arnazzini col titolo Mascherate bellissime da cantare il Carnevale.

È possibile che proprio la diffusione di una versione musicata abbia contribuito a promuovere la fortuna editoriale di questi testi, secondo un fenomeno già noto al repertorio

38

Bologna, Biblioteca dell'Archiginnasio. Ecco l'unico passo della dedica in questa sede degno di qualche interesse: “[...] per le molte cortesie, e favori, da lei ricevuti, fuora d'ogni mio merito, le vengo hora appresentare queste mie giocose Mascherate, per picciol segno di gratitudine, dalle quali spero, ch'ella sia per cavarne gustevole trattenimento, se non nella sufficienza del verso, almeno per le piacevoli, e varie inventioni di esse. [...]"

39 Se ne conosce una ristampa (Trevigi, Angelo Righettini 1624) da un manoscritto che la ricopia conservato presso l'Archiginnasio di Bologna.

40 È nota una edizione non datata e una ristampa del 1628.

41 Senza data. In tutti gli esemplari a me noti un listello con dicitura "Girolamo Cochi" è applicato sopra il nome dello stampatore originale.

42 Uno corrisponde comunque alla Mascherata di ortolane dalla Veglia carnevalesca, sopra trascritta. 
canzonettistico. Vale la pena di osservare che, con l'eccezione di quelle modenesi, tutte le edizioni citate continuano a riportare l'invito all'utilizzo canoro dei testi nell'ambito del carnevale. Di più, le edizioni delle Trenta mascherate recano sul frontespizio due diverse xilografie (figg.1-2) che ritraggono tre personaggi mascherati di cui quello centrale suona uno strumento musicale. Le illustrazioni delle citate ventarole non mostrano strumenti musicali ma le 'vedove' e i 'Pantaloni' raffigurati sono ugualmente in numero di tre, e l'idea che in ciò si possa leggere il riferimento ad un'esecuzione, e quindi a una composizione musicale, a tre voci appare suggestiva, per quanto ipotetica.

A fronte di una provata dinamicità della tradizione, l'importanza di identificare con sicurezza la specifica fonte letteraria utilizzata da Puliti, al fine di valutare motivazioni e criteri di selezione, estensione e dettagli degli interventi sul testo poetico, appare evidente. La precedente lista bibliografica non esaurisce ovviamente le fonti delle mascherate del Croce cui Puliti potrebbe avere avuto accesso: un'ulteriore edizione non nota, una copia manoscritta o anche una fonte musicale, come per gli altri testi della Ghirlanda. Tuttavia, allo stato attuale delle conoscenze, appare verosimile che Puliti abbia utilizzato l'edizione veneziana del 1603.

Rispetto all'edizione bolognese del 1604, l'edizione veneziana aveva naturalmente maggiori probabilità di giungere tra le mani di un lettore attivo tra Trieste, Muggia, Capodistria e Pola. La considerazione, di per sé certo non sufficiente, trova conforto nel confronto tra le due redazioni (d'ora in poi Venezia 1603 e Bologna 1604) e i testi riportati nell'edizione musicale (Puliti 1612). Le citate discrepanze a livello ortografico tra le due edizioni poetiche (punteggiatura, accenti, elisioni, troncamenti, apostrofi, uso delle maiuscole e, in minor misura, varianti lessicali) non consentono di stabilire la paternità di Puliti 1612 dal momento che anche nella redazione di quest'ultima sono intervenuti, a questo livello linguistico, analoghi meccanismi di alterazione più o meno casuale. Si veda quale esempio la seguente strofa dalla Creanza legata dai villani (mascherata n. 5 , terza strofa in Puliti 1612) dove, a fronte di una coerenza, in questo caso sicura, nell'uso delle maiuscole nelle due edizioni poetiche, l'edizione musicale offre una lezione promiscua:

\section{Puliti 1612}

Ch'io non fui si tosto entrata Frà le mandre, e frà gli ovili, Che da lor fui assaltata, Con Zappon, Vanghe, e Badili, Et altri ordegni

Vili, et indegni, Come fan fede i Villaneschi sdegni.

\section{Venezia 1603}

Ch'io non fui si tosto entrata Frà le Mandre, e frà gli Ovili, Che da lor fui assaltata, Con Zappon, Vanghe, e Badili, Et altri ordegni Vili, et indegni, Come fan fede i Villaneschi sdegni.

\section{Bologna 1604}

Ch'io non fui sì tosto entrata Frà le mandre, e frà gli ovili, Che da lor fui assaltata

Con zappon, vanghe, e badili, Et altri ordegni Vili, et indegni, Come fan fede i villaneschi sdegni.

Fortunatamente 3 mascherate mostrano nel dettato di alcuni versi discrepanze più rilevanti che lasciano pochi dubbi sulla discendenza di Puliti 1612 da Venezia 1603 piuttosto che da Bologna 1604. Così nella mascherata delle Malmaritate (n. 2 in Puliti 1612, strofa 5, versi 1-2 e strofa finale): 
MUZIKOLOŠKI ZBORNIK • MUSICOLOGICAL ANNUAL XXXVI

$\begin{array}{lll}\begin{array}{l}\text { Puliti } \mathbf{1 6 1 2} \\ \text { Ei se'n van co i loro amici } \\ \text { A cavar tutti i capricci,... }\end{array} & \begin{array}{l}\text { Venezia } \mathbf{1 6 0 3} \\ \text { Ei se'n van co i lor amici } \\ \text { A cavar tutti i capricci, ... }\end{array} & \begin{array}{l}\text { Bologna 1604 } \\ \text { Ei sen van co i loro amici } \\ \text { A cavarsi i lor caprici ... }\end{array} \\ \begin{array}{lll}\text { Mà perche potrian trovarci } \\ \text { Quì per strada a lamentarci } \\ \text { Ci vogliamo andar con Dio }\end{array} & \begin{array}{l}\text { Mà perche potrian trovarci } \\ \text { Quì per strada, à lamentarci } \\ \text { Ci volgiamo andar con dio, }\end{array} & \begin{array}{l}\text { Ma perche potrian trovarci } \\ \text { Quì per strada, a lamentarci, } \\ \text { Siam ciandiam con Dio }\end{array} \\ & \text { Siam vostre, à Dio. } & \text { Siam vostre, a Dio. }\end{array}$

Il caso della mascherata delle Spose contente (n. 4 in Puliti 1612, penultima strofa) sembrerebbe a prima vista deporre a favore di Bologna 1604, ma così non è:

\section{Puliti 1612}

Però cantando andiamo

In questa, e in quella parte,

Ch'il buon tempo c'habbiamo,

Tal gioia ne comparte ...

\section{Venezia 1603}

Però cantando andiamo

In questa, e in quella parte,

Il palese errore nel terzo verso in Venezia 1603 (che determina tra l'altro un'ipometria) venne evidentemente corretto sia in Bologna 1604 che in Puliti 1612; ma per qualche motivo la lezione originale errata venne mantenuta nella parte del Basso che recita: "Ch'il bon tempo habbiamo". Caso del tutto analogo nella Creanza legata dai villani (n. 5 in Puliti 1612, strofa 4):

\section{Puliti 1612}

Dopò havermi per villaggi Strascinata, e per le vie, ...

\section{Venezia 1603}

Dopò havermi per i villaggi

Strascinata, e per le vie ...

\section{Bologna 1604}

Dopò havermi pe' villaggi Strascinata, e per le vie, ...

dove la parte del Basso mantiene l'ipermetria di Venezia 1603.

Se ammettiamo che Puliti avesse a disposizione le 27 mascherate di Venezia 1603 piuttosto che una loro selezione (per esempio una raccolta di ventarole), è necessario individuare motivi e criteri che guidarono la selezione degli 8 testi. Ecco l'elenco delle mascherate nell'edizione del Croce dall'indice di Venezia 1603 (pp. 5-6), in grassetto quelle musicate da Puliti:

\section{Tab. 2}

1. Vedove che vanno piangendo i loro mariti morti (Puliti n. 1; 4/4)

2. Hortolane che portano insalatte, frutti, fiori et $\mathrm{c}$.

3. Balie che vanno cercando bambini da lattare

4. Todeschi fugiti de loro paesi per sospetto della guerra (Puliti n. 16; 7/7)

5. Donne mal maritate (Puliti n. 2; 8/13: 1-5, 9, 12-13)

6. Fanci[u]lli che menano Amor legato per la città

7. Spose contente che narrano la bontà de lor mariti (Puliti n. 4; 6/9: 1-3, 6, 8-9)

8. La Creanza legata da i Villani (Puliti n. 5; 6/10: 1, 3-4, 6, 8, 10)

9. Imitatrici da fiori di seta artificiosi 
10. Soldati svaleggiati che vengon di Ungaria (Pulitin. 14; 7/16: 1-2, 4, 6, 14-16)

11. Le Virtù che vanno cercando la Cortesia

12. Pantaloni innamorati (Pulitin. 9; 6/14: 1, 3-5, 7, 13/14)

13. Ciechi guidati d'Amore (Puliti n. 18; 4/4)

14. Facchini di Val brombana

15. Steccalegni che cercano zocchi da stellare

16. Scardassini da lana

17. Ministre di Venere che vanno cercando Amore

18. Gratiani

19. Cuciniere

20. Spazzacamini

21. Donne che vanno cercando da far bugate

22. I corrieri d'Amore

23. Formaggieri

24. Artigiani falliti

25. Ministre d'Amore

26. Pentolari

27. Contadini innamorati.

La concentrazione dei testi utilizzati da Puliti nella prima metà della raccolta sembrerebbe in effetti avvallare l'ipotesi che il compositore abbia avuto tra le mani una fonte parziale (es.: Le tredici mascherate piacevolissime ...). A rafforzare tale impressione, 3 delle 4 mascherate che vertono su soggetti presenti nella Ghirlanda (Graziani, spazzacamini, ortolani, falliti) ma i cui testi Puliti trasse da altre fonti (vedi tab. 1), sono localizzate nella seconda metà della raccolta.

L'analisi dei testi croceschi rende però plausibile una diversa conclusione. Esiste un'effettiva bipartizione della raccolta determinata dalla differente struttura dei testi poetici. Le mascherate dalla n. 14 alla n. 27 sono infatti monostrofiche: le numero 24 e 27 sono sonetti; negli altri casi si tratta di canzonette con una lunghezza che va dai 10 ai 21 versi e una struttura analoga a quella della Mascherata di ortolane che vendono latte dalla Veglia carnevalesca sopra riportata, dove la successione di endecasillabi e settenari e la struttura delle rime, pur con prevalenza della rima baciata, presentano irregolarità tali da impedire l'articolazione in strofe simmetriche, salvo un deciso intervento poetico di rimaneggiamento. ${ }^{43} \mathrm{Nel}$ repertorio musicale della canzonetta i testi monostrofici non sono certo rari ma l' $80 \%$ di essi non supera i 6 versi e non si danno casi in cui superino i 16. ${ }^{44}$ Sembrerebbe dunque che per i testi raggruppati nella seconda parte della raccolta in Croce l'ambizione poetica abbia prevalso sulla funzionalità musicale, ovvero che il poeta auspicasse un tipo di intonazione più raffinato, come suggerisce il fatto che nel citato passo della Veglia carnevalesca egli non usi il termine 'canzonette' bensì 'madrigali'. Puliti mostrò comunque di non gradire questi testi, predilegendo le forme polistrofiche della prima parte. Lo conferma lo scarto della Mascherata di ortolane (n. 2) la cui struttura rimica funge da incatenatura tra le terzine di cui è composta, rendendo anche qui impossibile un'articolazione in strofe che avrebbe dovuto essere necessariamente

${ }_{44}^{43}$ La prassi è attestata in casi di trasformazione di madrigali in canzonette. Si veda C. Assenza, op. cit., pp. 110 e sgg. Ibid., p. 143. 
selettiva vista la lunghezza del testo consistente per buona parte in una enumerazione di ortaggi.

Un altro tipo di forma poetica che non dovette incontrare il pieno gradimento del musicista è quella, obsoleta, della barzelletta di ottonari che accomuna le mascherate n. 3 , 6 e 11, tutte scartate. Le mascherate n. 4 e n. 10, pure 'in barzelletta', furono invece mantenute probabilmente in virtù della particolare vervelinguistica (n. 4) e della singolarità del soggetto (n. 10) già apprezzato da Banchieri. ${ }^{45}$

Certamente più difficile risulta individuare criteri legati al contenuto dei testi. Uno di essi potrebbe essere identificato nel carattere locale, bolognese, che si riscontra in almeno tre mascherate escluse: la n. 3 (Balie di montagna ${ }^{46}$ che dichiarano di provenire "sin dal Alpi Pistoiesi"), la n. 24 (Artigiani falliti con i loro cappelli verdi in capo all'uso di Bologna), e la n. 9 (Donne imitatrici di fiori di seta, attività fiorente nella città emiliana). Quest'ultima mascherata non sarebbe infatti stata scartata per motivi formali, presentando struttura identica o affine alle mascherate n. 1, 7 e 13 che vennero invece intonate. A prescindere dalla sua struttura formale, la mascherata $n$. 14 sarebbe comunque stata scartata per un motivo ancora differente. È questo l'unico testo in cui Croce indica il numero dei personaggi:

\section{Fachini di Valbrambana \\ Cinque Fachì nu sem \\ Vegnut chi lò Segnur, \\ Per si dol bel Pais de Valbranbana ...}

Dal momento che tale numero deve corrispondere a quello delle voci musicali e che la metrica non consente la sostituzione di 'cinque' con 'tre', il testo sarebbe risultato inutilizzabile. Anche qui il riferimento alle cinque voci conferma l'impressione che il Croce auspicasse per i versi della seconda parte (introdotta proprio dal presente testo) un destino più gratificante di una modesta intonazione canzonettistica a tre voci.

Un secondo livello di selezione del materiale poetico riguarda le strofe delle singole mascherate (cfr. tab. 2) ${ }^{47}$. Nel caso dei testi più corti, n. 1, 4 e 13, l'edizione della Ghirlanda conserva tutte le strofe, ma nei restanti cinque testi, che contano da 9 a 16 strofe, la selezione era inevitabile. Che non si trattasse tuttavia di un semplice problema di spazio disponibile sulla pagina stampata lo rivela il fatto che per le mascherate n. 2, 4, 5 resterebbe posto per un'ulteriore strofa, mentre ciò non accade per la mascherata n. 14 che, come già osservato, dovette riscuotere un particolare gradimento presso il compositore. La selezione appare tutt'altro che meccanica anche se mantiene in ogni caso la prima e l'ultima strofa (contenenti presentazione e congedo), e predilige spesso le strofe ad esse attigue. Una parziale eccezione si riscontra nella mascherata dei Pantaloni inamorai che in Puliti ricava la strofa conclusiva cucendo quartina iniziale e distico finale dalla penultima ed ultima strofa del Croce.

45 Vedi quanto più sotto osservato circa la selezione delle strofe per questi due testi.

46 Riporto in questo caso le intitolazioni, più estese, che precedono ogni testo.

$47 \mathrm{Vi}$ si indica sotto forma di frazione il numero delle strofe intonate rispetto a quelle originali e la posizione delle prime nella fonte poetica. 
Puliti, n. 9

Donca fie care, e belle

De la sentenza vù,

$S$ 'in questo le hà tort'elle

$O$ veramente nù;

\author{
Croce, n. 12 \\ Donca fie care, e belle \\ Dè la sentenza vù, \\ $S$ 'in questo le hà tort'elle, \\ $O$ veramente nù; \\ Elle à starne à soiar, \\ $\mathrm{E}$ nù à volerle amar. \\ Penseghe un poco sora \\ Vù c'havè'l cervel san, \\ E spende una mezhora \\ Per nù, perche doman \\ Tornarem sel ve par \\ A udirne sententiar.
}

Tornarem sel ve par

Complessivamente il trattamento dei testi selezionati appare straordinariamente conservativo se consideriamo che su 48 strofe poco più di una decina sono le discrepanze testuali di qualche rilievo, alcune delle quali probabilmente involontarie, come nella Mascherata di Pantaloni dove il verso tronco "Certi visetti d'or" è reso piano ("Oro") a scapito però della rima con "amor" e dove il verso "Quai semo quà arivaì diviene un goffo "Quì semo quà arrivaì. Accanto ad opportuni interventi correttivi del testo poetico originale (oltre ai casi già illustrati: "Ne cosa desiamo / da lor ch'in un momento noi l'habbiamo" (Spose contente) diviene, più sensatamente "Ne cosa desiamo / da lor ch'in un momento non l'habbiamo"; 48 "vanie" nella mascherata dei Tedeschiè correttamente riscritto "vaine" (da 'Wein' = 'vino'); i versi ipermetri "Noi siamo spose contente" (dalla mascherata omonima) e "Che ritrovarsi di lume in tutto privo" (Ciechi) sono regolarizzati in "Noi siam spose contente" $\mathrm{e}$ "Che trovarsi di luce in tutto privo"; etc.) non mancano interventi meno limpidi ("Vi chied[i]am per pietade / un pò di caritade" (Ciechi) mutato in "Vi chiediam perdone / un pò di caritade ") e casi di palese equivoco come quello della voce straniera "Edelman" (gentiluomo) dalla mascherata dei Tedeschiche, non compresa, è trascritta come una proposizione, "E del man", priva di senso. ${ }^{49}$

Una tale fedele rispondenza della trascrizione della Ghirlanda all'originale poetico ribadisce da un lato l'atteggiamento singolarmente compiacente del compositore nei confronti dell'opera poetica del Croce, dall'altro costituisce un'ulteriore autorevole conferma della probabile dipendenza di Puliti 1612 da Venezia 1603.

Quanto esposto relativamente all'aspetto testuale offre la possibilità di alcune considerazioni generali e conclusive sulla raccolta della Ghirlanda. La datazione delle fonti elencate, che videro la luce attorno agli anni 1601-1605, se pone un sicuro termine post quem induce anche a sospettare che Puliti possa avere quantomeno iniziato la composizione delle sue mascherate già attorno al 1605. È d'altra parte lo stesso compositore

48 In questo caso Venezia 1603 e Bologna 1604 coincidono e, di nuovo, la parte del Basso in Puliti 1612 riporta la versione originale della fonte poetica.

Il medesimo fraintendimento è nella citata edizione di M. Rouch. 
nell'esordio della dedicatoria a riferire: "Questa Ghirlanda che di varij fior quest'anni passati da scherzo tessei solo per mia ricreatione e diletto ...", ma il passo non è stato messo nel debito risalto da Ivano Cavallini ${ }^{50}$ per un errore di trascrizione che ha ridotto il riferimento cronologico ad un singolo "anno passato".

Va invece sicuramente confermato il giudizio espresso da questo studioso ${ }^{51}$ circa l'ispirazione padana (potremmo precisare emiliana) della raccolta, resa evidente dall'utilizzo dei testi poetici del Croce e di quelli già intonati da Banchieri, Vecchi, Nicoletti, e la conseguente assenza di elementi specificamente istriani auspicati dal citato studio di Ennio Stipčević. Il riconosciuto carattere di "esercizio intellettualistico" ${ }^{52}$ della raccolta, è espressione di un distacco dalla fonte rituale popolare e di un ripiegamento su un repertorio artistico preesistente che Puliti portò con sé dalla sua "terra d'origine". È ancora la dedica a rivelare come la raccolta fosse nata per un uso domestico, solipsistico e quasi snaturante nella sua destinazione strumentale ("sonandola tal volta nel Liuto, nella Cetra, et in altri strumenti"). ${ }^{53}$ Non a caso la Ghirlandaappare lontana da una funzionalità rappresentativa. Molti dei testi messi in musica non erano, originariamente, mascherate bensì villanelle rifunzionalizzate solo 'nominalmente' con la semplice apposizione del titolo, e un brano come, per esempio, Amor di cortigiana incostante conserva evidente il suo carattere 'lirico' piuttosto che 'rappresentativo'. Inoltre l'edizione non possiede nulla dell'apparato di indicazioni sceniche che correda le mascherate di Gabrieli (1601) o che introduce molte opere di Banchieri. Infine alcune caratteristiche dei testi musicali segnalate da Cavallini, quali il ricambio del trio vocale in almeno sette organici diversi, o il ricorso alla Augenmusik, ${ }^{54}$ inibiscono evidentemente l'ipotesi di una precisa ricerca di funzionalità rappresentativa. Vien fatto di pensare piuttosto, per richiamare in causa un modello caro a Puliti, all'astratto e colto 'teatro dell'udito' coniato da Orazio Vecchi.

\footnotetext{
P. 184.

Op. cit., p. 177.

Ibidem.

Contrariamente a quanto ancora recentemente ha sostenuto Ennio Stipčević, Gabriello Puliti. Ranobarokni skladatelju Istri, Zagreb, Sveta Glazba 1996, p. 12, nessun passo della dedicatoria è interpretabile come testimonianza di una esecuzione delle mascherate presso la dimora del dedicatario.

Op. cit., pp. 184, 186-187.
} 


\section{LE TRENTA}

MASCHERATE

PIA CEVO LISSIME

Di Giulio Cefare Croce,

Dalle quali pigliando IInuentioni, Apofono fare concerti dilettenoli, nel tempo di Carnouale.

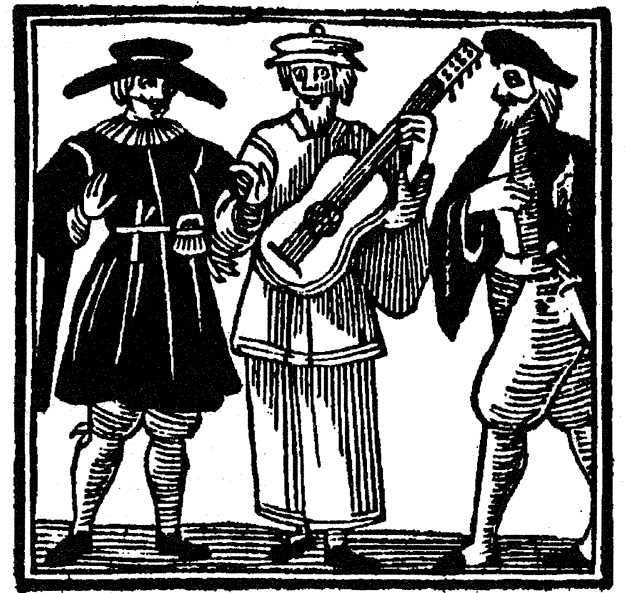

\section{In Bulogna per Girolamo Cacchis. Conlicuexededsperiori.}

Bologna, Biblioteca dell'Archiginnasio

\section{Appendice}

\section{Edizione delle rime di Giulio Cesare Croce messe in musica da Gabriello Puliti secondo Venezia 1603}

L'ordine delle mascherate è quello di Puliti 1612. Criteri di trascrizione: si è distinta la $u$ dalla $v$ secondo l'uso moderno; è stata unificata la grafia della $s$; E è stata trascritta et.

1.

Mascherata prima

Vedove che vanno piangendo $i$ loro mariti morti

Vedove sconsolate in bruna veste Tutte dolenti, e meste
Sospirando d'intorno

Andiam la notte, e ' 1 giorno

Pe' nostri fidelissimi mariti

Quai sono (ahi lasse noi) di vita usciti.

Perche essendo da loro abbandonate In assai fresca etate, 
Hora proviam ch'importe

Restar senza Consorte

Ne creduto havrian mai che patir tanto

Dovesse chi non hà Marito à canto.

Però voi, che dal Ciel vi vien concesso

D'haver marito appresso

Donne fatene conto

E con animo pronto

Siategli obedienti à tutte l'hore

Che perder il Marito è gran dolore.

Fede ne fanno i nostri pianti amari, Che i nostri à noi sì cari

Misere, persi habbiamo.

Ond'oltre che patiamo

Mille disagi, più ci preme, e duole

Dormir la notte in letto fredde, e sole.

2.

Mascherata quinta

Donne mal maritate, che vanno narrando le straniezze usatele da lor mariti

O che pena, ò che dolore,

O ch'affanno habbiamo al core

Noi meschine sventurate

Mal maritate.

Habbiam dato in certi humori, Bettolieri, e Giucatori, Che i dì intier ci fanno stare Senza mangiare.

Ci han giocato le Collane, E le vesti, e le Sottane, I Pendenti, con l'Anelle Ahi meschinelle.

Mà di più le Doti anchora Son andate a la mal hora; Deh mirate per pietade, Che crudeltade.

Ei se'n van co i lor amici

A cavar tutti i capricci,

E se noi pur guardiam fuori,

$\mathrm{O}$ che rumori.

[3 strofe omesse]
Tutto il mobil è finito,

E siam gionte à tal partito,

Che'l star vive habbiamo a nausa,

Per tal causa.

[2 strofe omesse]

Donne voi, che buon' gli havete

Date gratie al Ciel, che sete

Più di noi aventurate,

E furtunate.

Mà perche potrian trovarci Quì per strada, à lamentarci Ci volgiamo andar con dio, Siam vostre, à Dio.

4.

Mascherata settima

Le spose contente, che vanno narrando le bontà de lor Mariti

Noi siamo spose contente,

Donne come vedete,

Ch'andiam vezzosamente,

Cantando, allegre, e liete,

Poiche la buona sorte

N'hà dato à tutte quante un buon consorte.

La miglior compagnia, Che donna possa havere Habbiamo, e tuttavia Ci dan spasso, e piacere, Nè cosa desiamo

Da lor, ch'in un momento noi l'habbiamo.

I nostri buon Mariti

Mai non ci dan tormento, Mà son pronti, et uniti A darci ogni contento, E quel ch'aggrada, e piace A noi, ad essi ancor giova, e compiace.

[2 strofe omesse]

Se volessimo in fatto

Trar via la roba tutta, Ogni cosa è ben fatto, Ne alcun mai ci ributta, Mirate che bontade, 


\section{MUZIKOLOŠKI ZBORNIK • MUSICOLOGICAL ANNUAL XXXVI}

Donne gentil, dentro i lor petti cade?

[1 strofa omessa]

Però cantando andiamo

In questa, e in quella parte,

Ch'il buon tempo habbiamo,

Tal gioia ne comparte,

E la nostra allegrezza,

Procede solo, e vien da morbidezza.

Hor ci voglian partire

Da i vostri aspetti grati,

E tornar a gioire

Co i nostri sposi amati,

Dove fin ch'al Ciel piace

Vivremo insieme con amore, et pace.

5.

Mascherata ottava

La Creanza legata da villani et condutta per

la Cittade va facendo questo lamento

Oime Dio, chi mi soccorre

Da quest'empi, e rei villani.

Chi mi vienne, ahi lassa, à sciorre

Questi lacci iniqui, e strani,

Correte ò genti,

A miei lamenti,

E prendete pietà de miei tormenti.

[1 strofa omessa]

Ero gita per diporto

Questi giorni alquanto in villa,

Non pensando à simil torto,

Mà per star lieta, e tranquilla,

Mà son restata,

Ahime gabbata

Come vedete, e tutta mal trattata.

Ch'io non fui si tosto entrata

Frà le Mandre, e frà gli Ovili,

Che da lor fui assaltata,

Con Zappon, Vanghe, e Badili,

Et altri ordegni

Vili, et indegni,

Come fan fede i Villaneschi sdegni.

[1 strofa omessa]
Dopò havermi per i villaggi

Strascinata, e per le vie.

Et usati mille oltraggi

Mille stracij, e villanie,

Così legata

M'hà quì guidata

Di rustici instromenti circondata.

[1 strofa omessa]

E fra voi donne Gentili,

Vò tener mia nobil stanza,

Non frà genti inerme, e vili,

Che non san che sia creanza,

Ne pur han lume

D'un buon costume,

Send'usi fra le greggi, e al succidume.

[1 strofa omessa]

Poiche l'alma mia presenza,

Dal Villan poco si prezza,

Perche hà poca conoscenza

Di virtù, di gentilezza;

Mà i studij suoi

Son Capre, e Buoi,

E s'indiscreti son, miratel voi.

9.

Mascherata duodecima

Pantaloni innamorati, i quali narrano i loro

amori alle Gentildonne, facendole Giudicesse delle loro differentie

Vecchietti inamorai

Nù semo care Fie,

Quai semo quà arivai

Da vostre Signorie.

Per narrarve el brusor,

C'havemo dento al cuor.

[1 strofa omessa]

Nù amemo caldamente

Certi visetti d'or,

E brusemo talmente

Nel petto per so amor,

Che semo tutti fuogo,

E nò trovemo liogo. 
Gh'usemo servitue, Ghe femo sberretae, E le havemo tegnue D'ogn'hora appresentae $E$ in pe de guiderdon Le ne dà de murlon.

E ne dise chilosi, Balordi, et insensai, E Vecchi catarosi, E ne tien strapazzai Co se fossimo al fin Tanti asini, o fachin.

[1 strofa omessa]

No podemo magnar, Varde se havemo strette, Chel ne sconven pensar Sempre à ste mariolette, E farghe drio el corrier, Tiò, tiò, che bel piaser.

[5 strofe omesse]

Donca fie care, e belle Dè la sentenza vù, S'in questo le hà tort'elle, $O$ veramente nù; Elle à starne à soiar, E nù à volerle amar.

Penseghe un poco sora Vù c'havè'l cervel san, Espende una mezhora Per nù, perche doman Tornarem sel ve par A udirne sententiar.

14.

Mascherata decima Soldati svaligiati che vengono d'ungheria, $e$ domandano sussidio da poter andare ne lor paesi

Siam Soldati svalegiati, Che veniam de l'Ungheria, E siam stati per la via Da Nemici assassinati Siam Soldati [svalegiati].
Con lo Schioppo, e con la Spada

Fatto habbiam molte prodezze,

E pigliato assai Fortezze

Di quei Turchi rinegati, Siam Soldati [svalegiati].

[1 strofa omessa]

Acquistata habbiam Strigonia, Buda, Pappa, e Ghiavarino,

E levato un gran bottino,

D'oro, perle, e di Ducati.

Siam Soldati [svalegiati].

[1 strofa omessa]

Mà siam stati in certi boschi

Assaltati da Ladroni,

Quai con grossi e gran squadroni,

N'aspettavano à gli aguati. Siam Soldati [svalegiati]

[7 strofe omesse]

Horsu dunque almi signori, E voi Dame ornate, e belle, Allargate le scarselle, Che siam mezo desperati, Siam Soldati [svalegiati].

E con pronta, e larga mano Trate fuor Giulij, Carlini, Soccorrendo noi meschini, Che siam quì tutti affammati, Siam Soldati [svalegiati].

E quel poco che darete Pigliarem per caritade, E à la vostra nobiltade Restarem sempre obligati, Siam Soldati svalegiati.

16.

Mascherata quarta Todeschi che sono fuggiti $d^{\wedge}$ loro paesi per sospetto della guerra

Got morghen companie, $\mathrm{Nu}$ venir de nostre terre 
Per fuzir quel aspr guerre

Che far là per l'Ungarie,

Got morghen [companie].

Quand nu zunzer in Italie

$\mathrm{E}$ che guster stò bon vin,

$\mathrm{Nu}$ lassar nostr quattrin,

Prim zorn all'hostarie;

Got morghen [companie].

Se nu bever col bottaz,

Star aliegr not e zorno

Trinchi vanie và d'intorno

Con Tribian, e Malvasie,

Got morghen [companie].

Quattr cinqu' boccal d' vanie

Mai non basta a empir mie panze,

Botte piene n'è à bastanze

Da gonfiar budelle mie, Got morghen [companie].

Quand nu pò star aliegr Canter, rider, baller tant, Lassa pur Zorz galant'

Per le strade far pazzie

Got morghen [companie].

Car Fraù bel, e zentil,

Edelman car, e perfet

Se vu impir nostr flaschet,

$\mathrm{Nu}$ tenir per cortesie,

Got morghen [companie].

Horsù nu voler pregar, Che vu empir noster flascon,
Che nu star bon Compagnon;

E far brindes morghen frie,

Got morghen companie.

18.

Mascherata decimaterza

Ciechi guidati da Amore cantano isottoscritti versetti

Poveri ciechi siamo,

Che 'l lume perso habbiamo,

Sol per voler mirare

Troppo le luci chiare,

Donne Gentil, de vostri raggi ardenti,

Ch'acciecan non che abbagliano le genti.

Ahi che ben troppo arditi

Fussimo, mà invaghiti

Di quell'alma bellezza,

Ch'à Febo di chiarezza

Il pregio toglie, fummo tratti à forza

Che contra Amor non val humana forza.

Però da lui guidati

A i vostri aspetti grati

Vi chiedam per pietade

Un pò di caritade,

Che miseria maggior non hà l'huom vivo,

Che ritrovarsi di lume in tutto privo.

Moneta non vogliamo

Ne men pan vi chiediamo,

Mà sol udirvi dire,

Che del nostro martire,

Qualche dolor sentiate, che ciò grato

Ristor, fia al nostro miserabil stato. 
Dalle quali pigliando l' Inuentioni, fi poffono fare ce ncerti diletteuoli, egratiof. ael tempo di Carnouale.

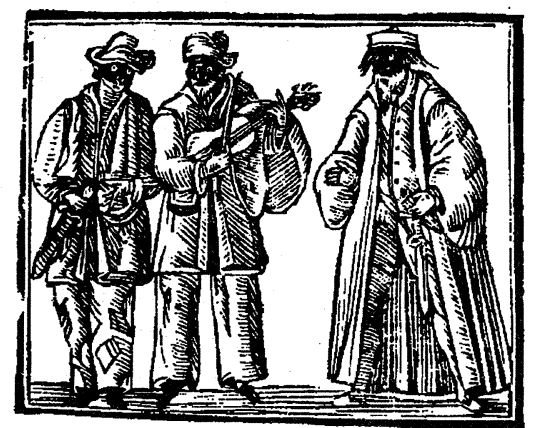

In Bologna, prefso gli Eredi del Cochi . 1628. al Pozzo rofso da S. Damizno.

Con licenza de' Superiosi.

Bologna, Biblioteca Universitaria

\section{Poetski izvori mascherat Gabriella Pulitija \\ Povzetek}

Doslej zelo nepopolno iskanje literarnih virov zbirke Ghirlanda odorifera (Dišeči venec; 1612) Gabriella Pulitija je obrodilo sadove šele v trenutku, ko je bilo mogoče ugotoviti, da skoraj vsa besedila izhajajo iz zbirke Mascherate (Maškarade; 1603) priljubljenega bolonjskega pesnika Giulia Cesara Croceja; ostala pa so znana iz že obstoječih zgodnejših tiskanih virov posvetne vokalne glasbe, tiskane vprvih letih 17. stoletja v Benetkah.

Razprava torej obravnava primer skladateljeve pogoste rabe poetskih besedil drugega avtorja, karje sicerv nemadrigalistični glasbeni literaturi redek primer. Po drugi strani lahko ugotovimo tudi to, kateri so glasbeniki in glasbeni viri, po katerih se je Puliti zgledoval pri sami izbiri besedil; poleg najbolj znanih imen, kot na primer Orazio Vecchi in Adriano Banchieri, omenimo še Filippa Nicolettija z zbirko Villanelle iz leta 1604. Podan je vpogled v Pulitijevo izbiro besedil in verjetnih skladateljevih posegih vanje ter ob spremembah, ki so bile namerne, pisec opozarja tudi na napake oz. nepravilna tolmačenja pri prepisovanju izvirnih besedil. Na podlagi datacije izvirnih izdaj lahko ne nazadnje domnevamo, da je Puliti zbirko Ghirlanda odorifera skladal na samem začetku stoletja, medtem ko jasna neizvirnost samih besedil padovanskih (v glavnem emilijanskih) avtorjev in virov, nedvomno izključuje možnost, da bi $v$ tej zbirki lahko videli izraz, pa četudi posreden, istrske kulture.

Prispevek malodane izključno obravnava literarno komponento Pulitijevih mascherat in se ne ukvarja $z$ odnosi med možnimi glasbenimi zgledi in skladateljevo glasbeno realizacijo. $V$ prilogi so navedena vsa besedila Giulia Cesara Croceja, ki jih je Puliti uglasbil. 